\title{
Connectivity, coverage and power consumption in large-scale wireless sensor networks
}

\author{
Q1 Hui Wang ${ }^{\mathrm{a}, *}$, H. Eduardo Roman ${ }^{\mathrm{b}}$, Liyong Yuan ${ }^{\mathrm{a}}$, Yongfeng Huang ${ }^{\mathrm{c}}$, Rongli Wang ${ }^{\mathrm{a}}$ \\ ${ }^{a}$ College of Mathematics, Physics and Information Engineering, Zhejiang Normal University, China \\ b ITCE, POSTECH, Pohang, South Korea \\ ${ }^{\mathrm{c}}$ School of Computing, National University of Singapore, Singapore
}

\section{A R T I C L E I N F O}

\section{Article history:}

Received 28 September 2013

Received in revised form 17 September 2014

Accepted 1 October 2014

Available online $\mathrm{xxxx}$

\section{Keywords:}

Wireless sensor networks

Connectivity

Coverage

Nodes switching off

Power control

\begin{abstract}
A B S T R A C T
Wireless sensor networks (WSNs) are typically constituted by a large number of connected sensors (nodes), generally distributed at random on a given surface area. In such largescale networks, the desired global system performance is achieved by gathering local information and decisions collected from each individual node. There exist three fundamental global issues on WSNs that we consider here, namely, full network connectivity, high coverage of the sensing area and reduced power consumption, thus improving on the network lifetime. Full connectivity can be obtained either by increasing the transmission range, at the expense of consuming higher transmission power, or by increasing the number of sensors, i.e. by increasing network costs. Both of them are closely related to global network lifetime, in the sense that the higher the power consumption or the more the number of active sensors present, the shorter the network lifetime (Wang et al., 2007) [1]. Here, we are interested in the minimal number of active nodes required for keeping the network functioning, while the problem of redundancy, i.e. having additional nodes kept in a sleeping mode for a certain period of time, can be implemented afterwards based on the present 'minimal' results. So the main question is, how can one design large-scale random networks in order to have both global connectivity and minimum number of active nodes reducing the total energy consumption? Although these questions have been addressed often in the past, a definite, simple predicting algorithm for achieving these goals does not exist so far. In this paper, we aim to discuss such a scheme and confront it with extensive simulations of random networks generated numerically. Specifically, we study the minimum number of nodes required to achieve full network connectivity, and present an analytical formula for estimating it. The results are in very good agreement with the numerical simulations as a function of transmission range. We also discuss results on how to further diminish network energy consumption by switching off some of the active nodes at random by keeping the connectivity of the whole network. The present results are expected to be useful for the design of more efficient WSNs.
\end{abstract}

(C) 2014 Published by Elsevier B.V.

\section{Introduction}

Wireless sensor networks (WSNs) are composed of elements (the sensors, also called nodes hereafter) that are

\footnotetext{
* Corresponding author.

Q1 E-mail address: hwang@zjnu.cn (H. Wang).
}

equipped with integrated functionalities of sensing, communication and computation [2-4]. The nodes typically operate with small batteries that are very difficult or expensive to recharge or replace, and can perform simple functions which may not be capable of implementing sophisticated coding, modulation and multiple access schemes [5]. These nodes are able to form a wireless 
network. They can communicate with their neighbors through wireless channels and with other nodes in the network through intermediate nodes, in order to coordinate their sensing activities. Furthermore, they can relay the data they sense to an specified data collection location, typically referred to as a sink.

WSNs are characterized by having a large number of connected wireless nodes [6]. Recent advances in MicroElectro-Mechanical Systems (MEMS), digital electronics and wireless communication technologies have enabled the deployment of large-scale WSNs, where thousands or even tens of thousands of nodes are distributed over a vast field to detect events of interests and send information to the sink [7,8]. Lack of any centralized control and the large-scale of network give rise to a number of challenging design and performance evaluation issues in such networks. Among them, a unique characteristic of WSNs is that the desired global system performance will be achieved based on local information and decisions collected from each individual node within the network.

One fundamental global property in WSNs is connectivity, ensuring that each node can transmit data to any other node over multiple hops [9]. It is a challenging task to maintain connectivity for the whole network because of the limited transmission range of sensors, due to power supply constraints, and due to the fact that some nodes may be down if they run out of energy. The latter can be prevented if an efficient working schedule is implemented. Connectivity is further affected when the wireless medium undergoes severe attenuation due to environmental (ambient) noise and external interference. Therefore, the connectivity among the nodes may vary with time.

Accordingly, the network topology can change rapidly and unpredictably over time, resulting in arbitrary topologies. In this case, the topology of WSNs may be determined by the transmission range, the scheduling scheme, and the characteristics of the wireless medium, while the control of the network is distributed among the nodes [10]. Consequently, it is a fundamental challenge for WSNs to maintain connectivity in a distributed fashion by the topology controlling functions that lie between the medium access control (MAC) and the network routing layer.

Network lifetime is inherently another fundamental global property; it is defined as the period from the start of network operation to the moment when the first node in the network runs out of energy [11-14]. One approach for network lifetime maximization is to reduce node's transmission power sufficiently in order to reach their farthest selected neighbor, which cannot only save energy but can also improve network throughput by mitigating the medium contention. Nevertheless, there is a price to be paid for this advantage because the shrinking of transmission range may strongly affect the network connectivity due to the decrease of the number of neighbor nodes connected to a given one (called node degree). Another effective means of conserving energy is to schedule nodes to sleep (i.e., turning off their radios) when they are not needed, without altering global connectivity and spatial coverage of the sensing field. The dependence of the connectivity on the transmission range and the number of running nodes greatly complicates analysis. In particular, how connectivity can be achieved by maximizing global network lifetime? While traditional theories, methodologies and techniques approaching this research problem are meaningful and efficient for the computations and analysis for small-scale networks, most of them are no longer applicable for large-scale networks due to the lack of scalability [15]. The interactions among large number of nodes and between different levels of the network architecture generate unexpected, often unpredictable global behaviors that need to be better understood.

To handle this question, in-depth analysis of the complex properties of WSNs are required. In particular, there is a need to measure the dependence of statistical global system properties of large-scale WSNs, such as average path distance, clustering coefficient, and degree distribution, on the network configuration, such as transmission range and the number of scheduling nodes, both qualitatively and quantitatively, for a better understanding of the intrinsic relations between them. Specifically, within different levels of the protocol structure of WSNs, the high-level (global) dynamical behaviors are not only determined by those at the lower (distributed and local) levels but also affected significantly by networking interactions.

In this paper, we assume that $N$ nodes are deployed in a surface region of given square area $L^{2}$. Packets are sent from node to node in a multihop fashion until they reach their final destination. Notice that here, we assume that there is only one sink node in the network. We study the statistical performance of the network controlled by one sink and derive the theoretical foundations of single-sink WSNs. The single-sink WSN results can be easily extended to multiple-sink networks by viewing the former as one component of the latter. Specifically, we consider Random Networks, where $N$ nodes are randomly located in space, i.e., independently and uniformly distributed in the plane. It is assumed that the nodes are homogeneous, i.e., all have the same transmission range or power.

We are interested in the following research problems: What is the degree distribution in such WSNs (in other words, distribution of the number of nearest neighbors)? The by-products are average degree and maximum degree. What is the average minimum number of nodes to keep the network connected within the given zone of area $L^{2}$ ? What is the average maximum number of removed nodes which still guarantees connectivity of the network, and what is the efficient scheduling scheme for removing them? How does the average minimum number of nodes change as the transmission range changes in the given area? What is the average shortest path distance? As a by-product, the network lifetime can be derived from that. What is the clustering coefficient? Answers to these questions provide important insights into the design and performance of large-scale WSNs to maintain the connectivity of the network while increasing its lifetime.

The main contributions of this paper are the following:

- We generate random sensor networks by deploying a minimum number of nodes required to achieve a fully connected network topology and essentially full area coverage. 
- We find an analytical expression for estimating the above average minimum number of nodes as a function of transmission range.

- We calculate several graph metric properties of the corresponding graphs suggesting different possible ways for getting further optimized networks.

- We derive the total power consumption of the network as a function of the number of nodes and disjoint sub-graphs whose nodes can be switched off alternatively by keeping full connectivity and coverage.

- We design the distributed management protocol to maintain both full connectivity and higher than $90 \%$ coverage in large-scale WSNs.

\section{Graph theory tools for WSNs}

To describe and characterize complex WSNs, we introduce some concepts and measures in complex networks which will be used in this work. Consider the setting on a planar square area $L^{2}$ and the following set of assumptions.

\subsection{Graph representation}

There are $N$ nodes randomly located within a square area $L^{2}$ on the plane. We assume that all nodes have the same transmission range, and have, in addition, the same sensing range equalling to the transmission range. Then, the resulting communication graph is called a unit disk graph (UDG), in which two nodes can communicate with each other directly if and only if their distance is smaller than the transmission range. The network can be represented by a set $V$ of nodes and a set $E$ of edges, linked together in a graph denoted as $G=(V, E)$. Therefore, the total number of nodes is $N=|V|$ and that of edges is $M=|E|$. Here, each edge is connected to one pair of nodes, one at each side. We consider the undirected network, in which edges have no orientation. In other words, any pair of nodes, such as $(i, j)$ and $(j, i)$ in the reverse ordering, are connected by the same edge.

\subsection{Average distance}

The distance between two nodes $i$ and $j$, denoted as $d_{i j}$, is equal to the total number of edges that connect them through shortest linkages. The diameter of the network, denoted $D$, is defined as the largest of all distances in the network; that is,

$$
D=\max _{i, j} d_{i j}
$$

The average distance of a network, or average shortest path, is defined as the average value of all distances over the network:

$$
\langle\ell\rangle=\frac{1}{\frac{1}{2} N(N-1)} \sum_{i>j} d_{i j}
$$

\subsection{Clustering coefficient}

If node $i$ has $k_{i}$ edges connecting it to other $k_{i}$ nodes, called neighbors of node $i$, it is easy to verify that there are at most $k_{i}\left(k_{i}-1\right) / 2$ edges among these $k_{i}$ nodes. Let $E_{i}$ be the number of the actual edges existing among the $k_{i}$ nodes. Then, the ratio between the real and the possible number of edges in the cluster of the $k_{i}$ nodes is defined as the clustering coefficient of node $i$, denoted as $C_{i}$; namely,

$C_{i}=2 \frac{E_{i}}{k_{i}\left(k_{i}-1\right)}$

\subsection{Degree and average degree}

In our setting, the degree of node $i$ in an undirected network is defined as the number $k_{i}$ of the edges connecting it to the other $k_{i}$ neighbors. It is intuitively clear that a node with higher degree will be "more important" than a one with lower degree in the network, because it will have more significant influence on other nodes and even the whole network, in terms of dynamics, information flows, data traffic, etc. Furthermore, the average degree of a network is the average value of all such node degrees $k_{i}$ over the entire network, denoted by $\langle k\rangle$.

\subsection{Degree distribution}

An important property of the network is the distribution of nodes with a certain degree. This degree distribution is defined by a probability distribution function, $P(k)$, which is the probability density that a randomly-picked node has degree $k$, where each node has an equal probability to be picked; that is,

\section{$P(k)=\{$ Probability that a node has degree $k$, the node is picked at random uniformly $\}$.}

\section{Related works}

Several previous works report results relevant to the present work such as network properties and maintenance techniques, including: connectivity, topology control, and power control for network lifetime maximization.

\subsection{Connectivity}

A basic concern in designing and implementing WSNs is connectivity to ensure that all of the nodes can be reached, which is a global property for constituting a connected graph. In this paper, we concentrate on connectivity and ignore wireless interference in the network because interference can be avoided through carefully scheduling transmissions. The undirected connectivity graph is used to support communication, where the set of nodes are defined as vertices and an edge $(i, j)$ is present if node $j$ is reachable by node $i$, and vice versa.

Based on arbitrary characteristics of WSN topology, the networks are typically modeled as random graphs $[16,17]$. Erdös and Rènyi (ER) random graph, $\mathcal{B}(N, p(N))$, is one of 
the most important random graph models consisting of $N$ nodes [18]. Given $N$ isolated nodes to start with, two nodes are chosen at random at each step, i.e., independently and identically, and then they are connected by an edge with probability $P(N)$, according to a uniform distribution. The critical probability for asymptotic connectivity of an ER random graph is given as follow [19]:

Theorem I. If we choose $p(N)=(\log N+c(N)) / N$, then the probability that $\mathcal{B}(N, p(N))$ is connected converges to one as $N \rightarrow \infty$ if and only if $c(N) \rightarrow+\infty$.

However, an ER random graph cannot detect the loss of connectivity in a network when the distance between nodes are larger than the transmission range. Random Geometric (RG) Graphs are more suitable for WSNs since the presence of a link between two nodes is based on the distance between the nodes [20]. Given $N$ nodes deployed in a plane at random, the edge is formed between two nodes $i$ and $j$ if and only if the distance between them is less than the transmission range $R(N)$. The transmission range $R(N)$ to obtain asymptotic connectivity of RG graph is given in the following relation $[21,22]$ :

Theorem II. If we choose $\pi R^{2}(N)=(\log N+c(N)) / N$, then the probability that $\mathcal{G}(N, R(N))$ is connected converges to one as $N \rightarrow \infty$ if and only if $c(N) \rightarrow+\infty$.

\subsection{Power control}

The global property of connectivity becomes very interesting in seeking local properties whereby the connectivity can be achieved. One such local properties is the transmission range and, assuming the reasonable noise and interference model, it translates into the number of neighbors of a node. The node can communicate with more neighbors by employing larger transmission range, but it costs more energy. Thus, minimizing power assignment for energy conservation has received much attention over the past few years [23].

An overview of power control is given in [24]. A centralized spanning tree algorithm for creating connected and bi-connected static networks with the objective of minimizing the maximum transmission power among all possible power assignments for each node, called the min-max power control, is described in [25]. Furthermore, two distributed heuristics are presented for mobile networks. The min-total power control is to obtain an optimal power allocation of total assigned power to all nodes among all possible power choices that can achieve a certain network property (e.g., the induced communication graph is connected). This problem has been shown to be NP-hard in [26]. An approximation algorithm with the same computational complexity as that of finding a minimum spanning tree has been suggested. Since then there has been much work in that problem and many approximation algorithms have been proposed with various network models, including symmetric links and asymmetric links, $k$-strongly connected and $k$-connected, bounded diameter, and $k$-disjoint paths.

\subsection{Topology control}

A wireless node can send the signal to any other node within its transmission range and communicate with the nodes beyond the range by using intermediate nodes to relay the message through multihop routing. From energy conservation point of view, each sensor should ensure that its transmission range is as low as possible and the network should ensure that the number of running nodes is as less as possible, both with the connectivity guaranteed. Edge can be added or removed when a node adjusts its transmission range or a node switches between sleep mode and wake-up mode. Topology control is to select either a subset of wireless devices or a subset of communication links that will be used for the network operations such as routing. Network topology control in large-scale energy-constrained WSNs becomes more complicated, since there is a tradeoff between "good" network property (i.e., maintain connectivity) and "efficient" energy saving (i.e., prolong network lifetime by adjusting transmission range and scheduling sleep periods for nodes). We can reconsider the above tradeoff in terms of network graph, a viewpoint which designs a subgraph of the original communication graph to ensure good network properties. Notice that not every connected subgraph of the UDG plays the same important role in network design.

Topology control is essential in order to maintain a connected topology among the nodes by restricting the number of networking nodes. It is required that any two nodes connected in a graph $G$ are also connected in the network topology. Since the topology forms the underlying network for routing protocols, it is also desirable that there exist energy efficient paths between potential source-destination pairs. Some metrics to describe the energy-efficiency, such as energy stretch factor and distance stretch factor, are defined in [27]. The lack of a fixed infrastructure in WSNs implies that such a computation on the network needs to be carried out in a distributed manner. Many distributed topology control algorithms have been proposed in [28-31]. In many routing algorithms, the network often represents a virtual backbone by establishing a subset of nodes, i.e., using the connected dominating set (CDS), which is often constructed from a dominating set or a maximal independent set [32-35]. The other imperative requirement for network topology control in WSNs is the fault tolerance. By setting the transmission range sufficiently large, the induced UDG will be $k$-connected without doubt. However, since long network lifetime is also required in WSNs, it is another challenging problem to find the minimum transmission range such that the induced UDG is multiply connected.

\subsection{Coverage}

One of the most active research fields in WSNs is that of coverage [36]. As pointed out in [37], the coverage concept is usually interpreted as how well a sensor network will monitor a field of interest. It can be thought of as a measure of quality of service and is subject to a wide range of interpretations due to a large variety of sensors and applications. The goal is to have each sensor located in the physical space of interest within the sensing range of 
at least one sensor. There are several factors which influence and restrict the operation of coverage in a WSN, depending on the particular applications and the capabilities of the deployed sensors.

The coverage provides several different concepts according to the particular application: Full or blanket coverage, target coverage and barrier coverage. (i) The coverage of an entire area is known as full or blanket coverage, which means that every single point within the field of interest is within the sensing range of at least one sensor. An important research problem is to find out how to deploy the minimum number of sensors within a field to achieve full coverage [38], where the concept of $r$-strip is used in order to locate each sensor at a distance $r$ away from the neighboring sensor. Here $r$ is the sensing range. (ii) Target coverage refers to observing a fixed number of targets. (iii) Barrier coverage refers to the detection of movement across a barrier of sensors. Energy saving is playing a key role in the development of all these coverage schemes.

There are two ways to deploy the sensors in the given area: Place them in predetermined locations or have them located at random. The deterministic deployment is a simple approach but, in many deployments, it is impractical. A more sophisticated deterministic way is given in [39], where arranging the sensors in a diamond pattern, corresponding to a Voronoi polygon, is proposed. However, while the deterministic approach has the advantage of simple deployment, it is not a practical design principle because of several limitations, including the sensing and communication ranges of every node being perfectly isotropic, as well as the ability to place the sensors in exact locations. Thus, random deployment is preferable in terms of practical requirements.

The concept of $k$-coverage is introduced to consider the application of multiple sensor monitoring each point in the field of interest, where $k$ represents the number of nodes that watch each point. The question to preserve $k$-coverage while minimizing the energy expended in the sensor nodes is addressed in [40]. In [41], a $k$-coverage maintaining scheme is proposed. When a node detects adequate $k$-coverage in the area it will put itself into a sleep mode. It will enter the wake-up mode after a random period of time and perform another check. The practical protocol by assigning to the nodes five different states, instead of just two, is proposed in [42]. The redundancy protocol by scheduling the redundant sensors with the lowest energy levels to turn off is proposed in [43]. The distributed algorithms for connected $k$-coverage is proposed in [44].

The concept of the Voronoi diagram is used gradually in the area of coverage computation. The concept is simple but intuitively appealing. Given a finite set of distinct and isolated points in a continuous space, all the locations in this space are associated with the closest member of the point set. Thus, the space is partitioned into a set of regions [45]. Although many researchers in the sensor network area have mentioned the critical notion of coverage in the network, this is the first time, to our knowledge, that an algorithmic approach combined with computational geometry constructs is adopted in large-scale wireless sensor networks. In this area, [46] was the first to identify the importance of computational geometry and Voronoi Diagrams in sensor network coverage. Our main concern in this paper is to provide full or blanket coverage in a WSN in a very efficient way.

\section{Complex networks approach: analytical results}

We consider a flat square zone of area $L^{2}$, lacking of any obstacles inside it, denoted as sensing area (SA), on which we can deploy $N$ identical wireless sensors at random so that their surface density is,

$\rho=\frac{N}{L^{2}}$

For our present purposes, and for the sake of simplicity, we assume that all sensors are equal, having the same transmission range and sensing range, denoted as $R$. Thus, we have only one parameter in our model, condition that can be relaxed later within a two parameters variant of the model, in a similar spirit as done for a type of a continuum percolation model [47]. The latter will be considered in a different work, being outside the scope of the present study.

Next, we construct a random sensor network in the following way. First note that two sensors are connected if they are located within a distance $R$ from each other. We start deploying sensors one-by-one at random and at each step we determine whether there exists a singly connected network of sensors and the corresponding SA coverage exceeds a lower cutoff, which we take to be $90 \%$. Specifically, we stop dropping sensors when full connectivity of the sensor network and at least 90\% coverage of the SA is reached for the first time. Typically, almost full coverage (i.e. about $99 \%$ ) is actually achieved.

It is further assumed that the transmission range is much smaller than $L$, which is generally the case in most applications. Thus, for simplicity, we derive our analytical predictions by neglecting boundary effects, that is, for the case of an effective infinitely large SA. The numerical results we present here, however, are for the case of finite sizes where boundary effects do play some roles, as we will see in particular situations.

Here, we are interested in obtaining a realistic estimate of the minimum number of sensors $N$ required to get full connectivity of the SA, as follows:

Theorem III. The mean number of nodes $N$ for getting full connectivity, for a fixed transmission range $R$ and fixed $S A L^{2}$ obeys, when $(R / L)^{2} \ll 1$,

$\frac{\pi R^{2}}{L^{2}}=\left(2 \ln N+\ln \left(\pi R^{2} / L^{2}\right)\right) \frac{1}{N}$.

Proof. Let us consider first a circular area of radius $R$ fully located inside the SA. A sensor dropped at random will hit this circular region with probability $p=\pi R^{2} / L^{2}$. Next, if $N$ sensors are dropped at random, the probability to find exactly $n$ sensors, called $P(n \mid N)$, within the same small circular area is given by the binomial distribution, i.e. $P(n \mid N)=\left(\begin{array}{c}N \\ n\end{array}\right) p^{n}(1-p)^{N-n}$. By taking the limit $N \gg 1$, which is the case which we are interested in here, $P(n \mid N)$ becomes the Poisson distribution,
469 


$$
P(n)=\frac{\left(\pi R^{2} \rho\right)^{n}}{n !} e^{-\pi R^{2} \rho},
$$

where the mean number of sensors located within the circular region is,

$$
\langle n\rangle=\pi R^{2} \rho \text {. }
$$

In other words, there are on average $\langle n\rangle$ sensors inside an area $\pi R^{2}$ located anywhere within the SA. In Eq. (3), the variable $N$ does not appear explicitly as an argument of $P(n)$ but it is contained implicitly in the density $\rho$.

Assume now there are $N$ sensors already deployed within the SA, and just one of them is still disconnected from their counterparts. This means that within a distance $R$ from the isolated sensor there no others present, event which has a probability of occurrence equal to $P(1)$. Note that $P(1) \rightarrow 0$ when $N \rightarrow \infty$. Since by hypothesis there is only one isolated sensor, the probability of such an event out of $N$ is just $1 / N$. Thus, we argue that $P(1)$ is of the order of $1 / N$ and the connectivity 'transition' we are looking for occurs when $P(1)=1 / N$. Using the expression for $P(1)$ according to Eq. (3), we find that the minimum number of nodes $N$ required to get a fully connected network is given by,

$N=\frac{L^{2}}{\pi R^{2}} \ln \left(\pi \frac{R^{2}}{L^{2}} N^{2}\right)$.

By expanding the logarithm on the right hand side of the equation and rearranging the different terms we obtain Eq. (2).

Our above result for $N$, Eq. (2), can be compared with the one in Theorem II, where there is a factor 2 difference in the logarithmic term on the right-hand-side of our equation, and the constant $C=\ln \left(\pi R^{2} / L^{2}\right)$ in our case does not depend on $N$. Our result Eq. (2) actually represents a (non-rigorous) estimate for the mean value of $N$ required for obtaining full connectivity and becomes more accurate the smaller the value of $R$ is, i.e. when $R \ll L$. This new result differs from the one discussed in Theorem II above (where $L$ is taken as unity, see [21,22]) in the fact that $R$ is constant here, independent of $N$. Our result Eq. (2), or equivalently Eq. (5), is more useful in practical calculations as it deals with a constant transmission range $R$, for a finite number of nodes $N$, on arbitrary length scales $L$.

Once full connectivity has been achieved we evaluate the properties of the associated graph according to the quantities discussed in Section 2. As mentioned there, a sensor is called a node in the graph and its number of connected nodes its degree, denoted as $k$. In particular, we can obtain the mean node degree, $\langle k\rangle$, for our network from the obvious relation,

$\langle k\rangle=\langle n\rangle-1$.

Therefore, the node degree distribution is also a Poisson distribution given by,

$P(k)=\frac{\langle k\rangle^{k}}{k !} e^{-\langle k\rangle}$.

In the following, we proceed to validate our theoretical prediction Eqs. (5) and (7).

\section{Numerical results: validation}

In the following, we discuss results for $L=500 \mathrm{~m}$ and consider different transmission ranges in the interval $R=(10,20,30,40,50,60,70,80) \mathrm{m}$. As explained above, we deploy sensors at random within the square area till full connectivity (every sensor is connected to the entire network) and at least $90 \%$ coverage are obtained. That is, there exists a single fully connected structure in the field covering almost the whole SA. An example of such a network is illustrated in Fig. 1(top panel) for the case $R=80 \mathrm{~m}$.

Since the procedure we have chosen is not optimized it may overestimate the number of nodes actually needed for getting full connectivity and coverage. We have therefore considered a simple algorithm to reduce the number of nodes already deployed on the SA by deleting (switching off) some of them. To this end, we pick a node at random and delete it if full network connectivity and above $90 \%$ coverage are maintained. If the deletion of a node breaks one of the above two conditions, the node is kept on and the deletion process is finished. This algorithm is adopted in keeping with a simple approach aimed at avoiding a full, more time consuming network analysis. The question is
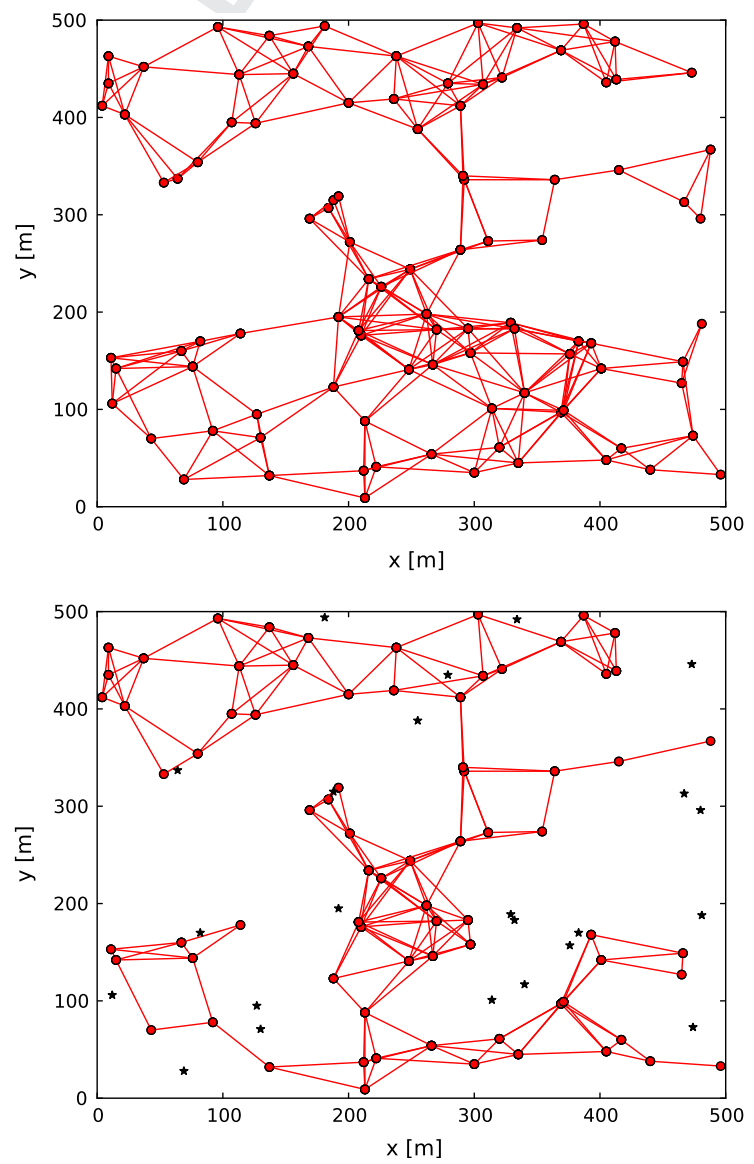

Fig. 1. Example of the original network for $R=80 \mathrm{~m}$ (top panel) and for the case in which some nodes have been deleted (switched off) (lower panel). 
whether such a random method proves to be useful in practice. As a result of the simulations, we find that typically, we can delete about $10 \%$ of nodes in this way, which is a rather good starting point for future improvements if necessary. In the lower panel of Fig. 1, we show the resulting network where about 20 nodes have been deleted from the original graph shown in the upper panel.

Based on these two types of networks, the original and the optimized one, we analyze some of their metric properties as discussed next. Let us consider first the mean number of nodes $N$, averaged over several realizations of the network, for the two above defined networks, the original and the optimized one. The resulting values of $N$ are displayed in Fig. 2 as a function of the transmission range, and compared with the theoretical prediction Eq. (5). Clearly, the optimized network has a lower number of active nodes (open circles), as compared to the original network results (full circles). Fig. 2 shows that despite the deletion process, the remaining number of sites is still well described by the theoretical result Eq. (5). As is apparent from the figure, the analytical estimation for the minimum number of nodes to get full connectivity works very well, in particular for small $R$. The prediction Eq. (5) should thus be useful in practical circumstances for estimating the minimum number of nodes required for connectivity.

For both the original network and the one with node deletions we calculate the mean shortest path and mean clustering coefficient, which are shown in Fig. 3 as a function of transmission range $R$. While the shortest path increases a bit for the deleted nodes case with respect to the original network, the corresponding change (decrease) in the clustering coefficient is more pronounced, as one can see from Fig. 3. This means that the global topology of the network remains essentially intact, while larger changes are found locally, as measured by the clustering coefficient.

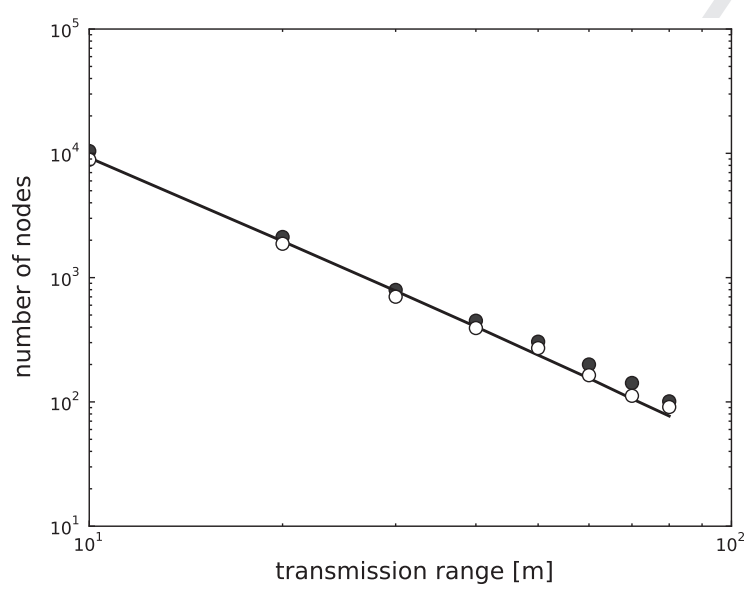

Fig. 2. Minimum mean number of nodes required to get full network connectivity as a function of transmission range $R[\mathrm{~m}]$. The numerical results are represented by the full circles and the prediction Eq. (5) is displayed by the continuous line. The open circles represent the results after some nodes have been deleted (switched off), but keeping the full connectivity and more than $90 \%$ coverage of the network. Here, we have used $L=500 \mathrm{~m}$. The error bars of the points amount to a $14 \%$.
This result suggests a possible way for getting even more efficient networks, exploration which we are going to discuss below.

Let us consider now the issue of the distribution of node degree. Node degree distribution functions have been calculated in each case, and few representative examples are displayed in Fig. 4. As we can see from this figure, the node degree distributions are consistent with the Poisson prediction Eq. (7), except for small deviations occurring at small degree $k$. The latter are due to boundary effects, being more important for large transmission ranges, as can be expected.

Our final results are summarized in Table 1 for the original network and in Table 2 for the network with deletions. Averages have been performed over about 50 configurations in each case.

\section{Optimized networks: management protocols}

In this section, we discuss how to further optimize the choice of sensors which can be switched off by keeping both full connectivity and higher than $90 \%$ coverage. From this procedure a method for networking management designed to diminish energy consumption of the whole network is presented.

\subsection{Centralized management scheme: disjoint sub-graphs}

In Section 5, we show that generally one can delete an appreciable number of nodes while still keeping full network connectivity and large coverage. Here, we discuss a systematic method in order to find all possible nodes that can be switched off with the same constraints.

The idea is very simple and consists in scanning all nodes, taking at random or sequentially, and assigning an index to each node which has already been selected for deletion. If the node has already been selected it cannot be considered again. In this way, the new node selection is performed on a smaller number of nodes and the process converges very fast to its end. Every time a node is

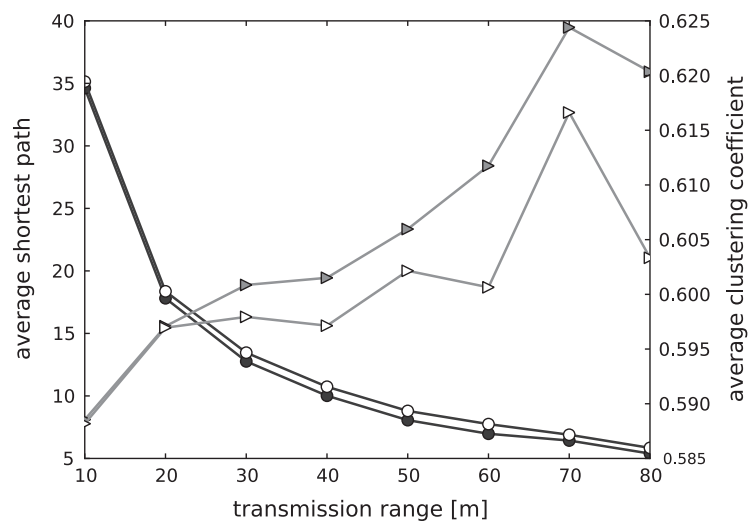

Fig. 3. Average shortest path (circles) and clustering coefficient (triangles) as a function of transmission range $R[\mathrm{~m}]$. The full symbols represent the results for the original network, the open ones for the optimized one. 

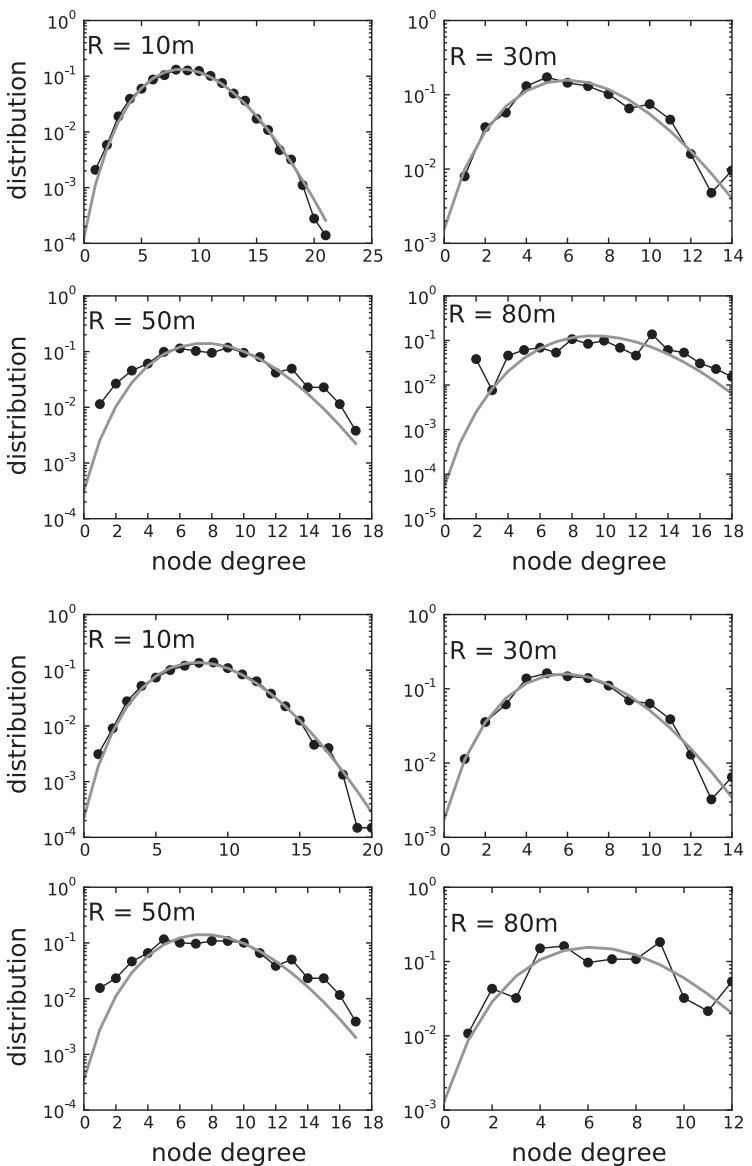

Fig. 4. Node degree distribution functions as a function of node degree $k$ for different transmission ranges $R[\mathrm{~m}]$. Top panel: original networks. Lower panel: networks with node deletions. selected, it is checked whether connectivity and coverage criteria are satisfied if the node is switched off, and if yes, then it is deleted, otherwise it is kept on. At the end of the procedure, the working part of the network is built from the nodes remained on, while the remaining nodes get switched off. For the latter, we denote their number as $S_{1}$, where the subindex 1 indicates the first optimization step, $i=1$.

The process continues in the following way. The $S_{1}$ nodes which were off at the first step are turned on and cannot be selected again for being switched off. Then, we starts again switching off nodes among the remaining $N-S_{1}$ nodes. In the second optimization steps, $i=2$, we find $S_{2}$ which can be switched off without altering full connectivity and coverage. The set of the $S_{2}$ is different than the set of $S_{1}$ nodes. We repeat the process till all nodes have been switched off at least once. Let us denote the final optimization step by $n$. It is clear that $S_{i}$ obeys the following sum rule, $\sum_{i=1}^{n} S_{i}=N$.

Results are shown in Table 3 for the case $R=80 \mathrm{~m}$ and for two realizations of the network, one containing originally $N=175$ nodes and a second case in which $N=90$ nodes. As one can see, the larger $N$ is the smaller the total number of optimization steps becomes. For $N=175, n \cong 2$. This means that there are two disjoint sub-graphs in the network which can be switched off alternatively. For $N=90$, however, $n \cong 10$ and there are more sub-graphs to consider. Of course, since in the latter the number of nodes is already small, it is more difficult to switch off nodes than when their initial number is large. Here, small and large are referred to the average value described by Eq. (5). For $R=80 \mathrm{~m}$, the mean number is $\langle N\rangle \cong 106$.

Illustrative examples of the original graphs and its subgraphs are shown in Figs. 5-7 for $R=80 \mathrm{~m}$. As one can see,
680

Table 1

Graph properties of wireless sensor networks for: transmission range $R[\mathrm{~m}]$, ratio $R / L$, mean shortest path, mean clustering coefficient, average node degree $\langle k\rangle$, standard deviation of node degrees over different configurations, mean maximum degree in the network.

\begin{tabular}{llllllll}
\hline$R$ & $R / L$ & Nodes & Shortest path & C-coefficient & Average degree & S.D. & $K_{\max }$ \\
\hline 10 & 0.02 & 10,426 & 34.62 & 0.589 & 12.96 & 10.25 & 1.774 \\
20 & 0.04 & 2122 & 17.81 & 0.597 & 8.504 & 2.300 \\
30 & 0.06 & 796 & 12.76 & 0.601 & 8.448 & 1.796 \\
40 & 0.08 & 450 & 10.02 & 0.602 & 8.742 & 1.777 \\
50 & 0.10 & 303 & 8.062 & 0.606 & 8.195 & 18.1 \\
60 & 0.12 & 200 & 6.974 & 0.612 & 7.249 & 2.463 \\
70 & 0.14 & 132 & 6.462 & 0.624 & 1.687 \\
80 & 0.16 & 106 & 5.389 & 0.620 & 7.441 & 16.0 \\
\hline
\end{tabular}

Table 2

same as in Table 1 for 'optimized' wireless sensor networks with deletions.

\begin{tabular}{|c|c|c|c|c|c|c|c|}
\hline$R$ & $R / L$ & Nodes & Shortest path & C-coefficient & Average degree & S.D. & $K_{\max }$ \\
\hline 10 & 0.02 & 7421 & 35.139 & 0.588 & 9.227 & 1.080 & 22.2 \\
\hline 20 & 0.04 & 1873 & 18.363 & 0.597 & 9.050 & 1.455 & 20.4 \\
\hline 30 & 0.06 & 702 & 13.460 & 0.598 & 7.462 & 1.204 & 16.1 \\
\hline 40 & 0.08 & 393 & 10.735 & 0.597 & 7.369 & 1.460 & 16.8 \\
\hline 50 & 0.10 & 271 & 8.800 & 0.602 & 7.824 & 2.568 & 16.4 \\
\hline 60 & 0.12 & 164 & 7.747 & 0.601 & 6.714 & 1.298 & 14.1 \\
\hline 70 & 0.14 & 112 & 6.894 & 0.617 & 6.072 & 1.134 & 12.2 \\
\hline 80 & 0.16 & 91 & 5.844 & 0.603 & 6.366 & 1.750 & 12.3 \\
\hline
\end{tabular}


Table 3

Disjoint sub-graphs: $N$ is the number of nodes of the original graph, $\langle n\rangle$ is the mean number of disjoint graphs averaged over 20 realizations of the random search, $\langle S\rangle$ is the mean number of deleted nodes (switched off), and $\left\langle S^{2}\right\rangle$ its second moment, and $\sigma$ is the s.d. of $S$, for $R=80 \mathrm{~m}$.

\begin{tabular}{rrrrr}
\hline \multicolumn{1}{c}{$N$} & $\langle n\rangle$ & $\langle S\rangle$ & \multicolumn{1}{c}{$\left\langle S^{2}\right\rangle$} & \multicolumn{1}{c}{$\sigma$} \\
\hline 90 & 10.55 & 8.5 & 113.1 & 6.39 \\
175 & 2.85 & 61.4 & 4842.3 & 32.75 \\
\hline
\end{tabular}

the graphs are fully connected, and the coverage (white zone) is large. Additionally, the node degree distribution remains Poissonian to a large extent. Further results are displayed in Table 4 for the mean shortest path, clustering coefficient and average node degree. The former increases as the number of deleted nodes increases and the clustering coefficient does not change appreciably, except when the number of deleted nodes becomes large. Finally, the mean node degree decreases sharply as the number of deleted nodes increases, as one can expect.

\subsection{Performance evaluation}

According to the results of the previous section we can implement a management protocol for switching on and off nodes. In the protocol, the set of nodes indicated by the numbers $S_{i}$, are switched off sequentially, thus allowing the corresponding sensors to stay idle for a period of time, which we denote as $\tau$, prolonging its life span. The following result regarding energy consumption of the network can be derived for this algorithm.

Theorem IV. The total power consumption of a random sensor network consisting of $N$ nodes, displaying full connectivity according to Theorem III and composed of $n$ disjoint sub-graphs, where each sensor requires a power $w$ for its functioning, is given by

$W_{\mathrm{n}}=w\left(1-\frac{1}{n}\right) N$
Proof. Assume we turn on one sub-network (out of $n$ ) at a time, by keeping the remaining ones off, during say an interval of time $\tau$. Once all associated sub-graphs have been considered, the total elapsed time or full working period $T$ of the network has been reached, which is just $T=n \tau$. During each time interval $\tau$ there are $S_{i}$ nodes off, with $1 \leqslant i \leqslant n$, and therefore the energy consumption becomes $E_{i}(\tau)=w \tau\left(N-S_{i}\right)$. Then, using the fact that $N=\sum_{i=1}^{n} S_{i}$, the total energy consumption during the full working period $T$ becomes, $E_{\mathrm{T}}=\sum_{i=1}^{n} E_{i}(\tau)=w \tau(n-1) N$, which can be written in terms of $T$ as,

$E_{\mathrm{T}}=w T\left(1-\frac{1}{n}\right) N$

Thus, the whole network power consumption is $W_{\mathrm{n}}=E_{\mathrm{T}} / T$, and Eq. (8) is obtained.

The present method, based on the concept of subgraphs which can be set on and off alternatively, can be generalized using the concept of redundancy, as discussed in the abstract. Redundant subgraphs can be easily implemented within this scheme once the minimum number of nodes required to maintain full network connectivity and coverage is known.

\subsection{Distributed management scheme}

In our proposed scheme, the neighboring set of node $i$, denoted by $N B(i)$, is defined as the set consisting of neighboring nodes, denoted generically as $n b$, as well as the node itself. For example, consider the small network in Fig. 8. The neighboring set of node $a$ is the set of $\{a, b, c, d, e, f\}$, which is the same as for node $b$ 's. The set that consists of the nodes with the same NB is called redundant set, denoted by $R D$. In the above network example, the $R D$ includes nodes $a$ and $b$. It is obviously apparent that the distance between nodes with the same NB is smaller than their transmission range. This kind of nodes has the potential to work alternatively, one on and the other off, without breaking the connectivity and coverage conditions in the network. Further, the claim has been validated by our simulation experiments. However, one of key problems here is
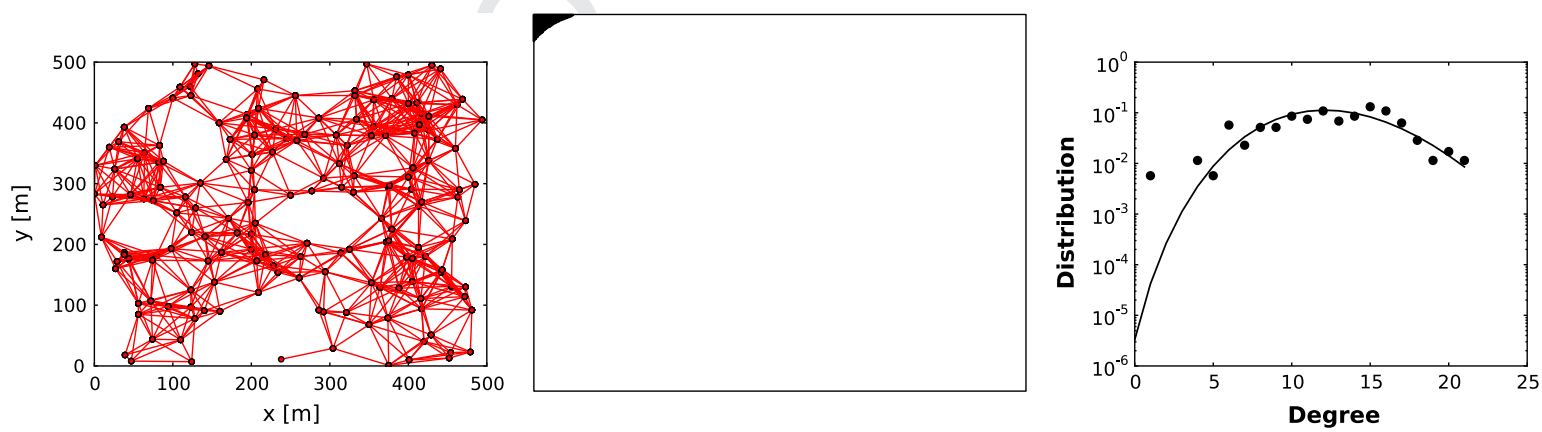

Fig. 5. Original network (left panel): $(N=175$ nodes). Coverage (central panel): uncovered sectors represented by the black patches. Node degree distribution (right panel). 

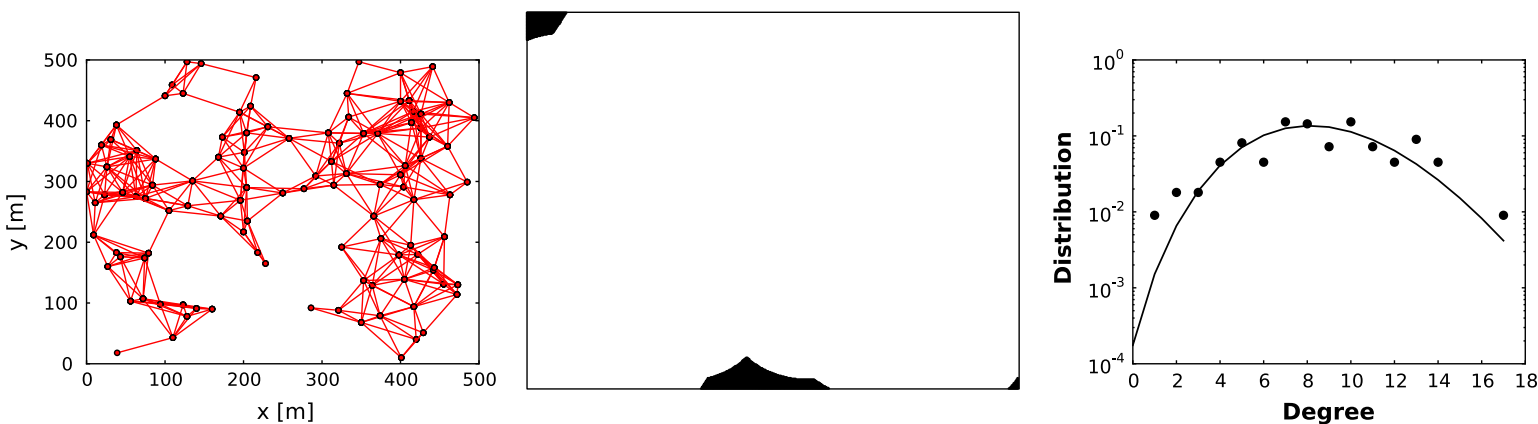

Fig. 6. Same as in Fig. 5 for the first subnetwork $(N=111$ On-nodes $)$.
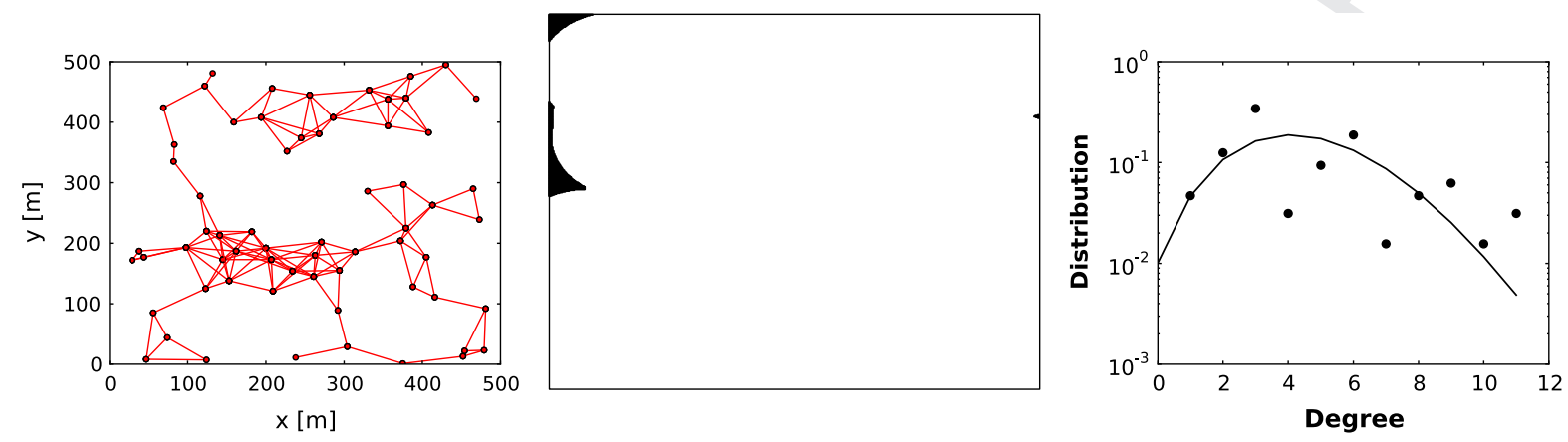

Fig. 7. Same as in Fig. 5 for the second subnetwork ( $N=64$ On-nodes).

to find all the $R D$ s in the network and design a scheme to coordinate and schedule the nodes in the $R D$ to alternate between sleep and work.

To find the RDs in the network by their own, each node shares its neighboring information with its neighboring nodes, so that each node will know the neighboring information of its two-hop neighboring nodes. The nodes in the $R D$ attempt to coordinate and synchronize sleep schedules by periodic (to compensate for clock drift) exchanges of nodes' schedules. At each time, only one of them is selected as the agent of $R D$ to work, denoted by $A R D$. The algorithm for discovering the $R D$ s is discussed as follows ${ }^{1}$

Algorithm 1. Find $R D$.

$$
\begin{aligned}
& R D=0 \\
& \text { for all } n b \in\{N B(i)-i\} \text { do } \\
& \text { if } N B(n b)=N B(i) \text { then } \\
& \quad R D:=R D \cup n b \\
& \text { else if } N B(n b)=2 \text { then } \\
& \quad R D:=n b \\
& \text { end if } \\
& \text { end for } \\
& \text { return } R D
\end{aligned}
$$

\footnotetext{
${ }^{1}$ We use the notation " $a=b$ " when we are asserting a statement of fact, that the value of a is equal to the value of $b$. In contrast, we write " $a:=b$ " to denote an operation in which we set the value of a variable $a$ to be equal to the value of $b$. In other words, this operation overwrites $a$ with the value of $b$.
}

In our proposed distributed management protocol, the indicator function (IF) is used to select $A R D$ as follows

$$
I F(i)=\frac{E r_{i}}{|N B(i)-i|} \prod_{j \in N B(i)-i} L(i, j)
$$

where $i$ denotes a node in the network and $j$ represents the $n b$ in the neighboring set $N B(i)$ of node $i$. $E r_{i}$ denotes the remaining energy of node $i$. $L(i, j)$ represents the quality of link between the node $i$ and $j .|\cdot|$ denotes the cardinality of the set.

To make the nodes consume their energy evenly, the nodes in a $R D$ will be selected as the $A R D$ one after the other, in such a way that each node will act as such individually for a given amount of time, which is taken the same for each node. In the first round, the node with higher IF than the given threshold announces itself as the ARD by broadcasting the messages within its $N B$. In the message, its address is stored at the bit of "ARD". When the $n b s$ in its $N B$ receive the messages, they update their $N B$ list and the bit of $A R D$. If the node that has not been selected as $A R D$ finds its $I F$ higher than that of the current $A R D$, it will announce itself as the $A R D$ in the next round. If the current $A R D$ realizes that no node can take its place within the given circle, it will announce itself as the $A R D$ again in the next round.

\subsection{Simulation experiments}

The distributed management protocol is applied to the problem of distributed schedule of the nodes in the $R D s$ 
Table 4

Statistical properties of the original network and of its disjoint graphs, $N=175$ and $R=80 \mathrm{~m}$. Displayed are the mean shortest path, clustering coefficient and mean node degree.

\begin{tabular}{rlll}
\hline \multicolumn{1}{l}{$N$} & Shortest path & Clustering coefficient & Node degree \\
\hline 175 & 4.41 & 0.63 & 12.62 \\
111 & 5.01 & 0.65 & 8.65 \\
64 & 7.24 & 0.54 & 4.59 \\
\hline
\end{tabular}

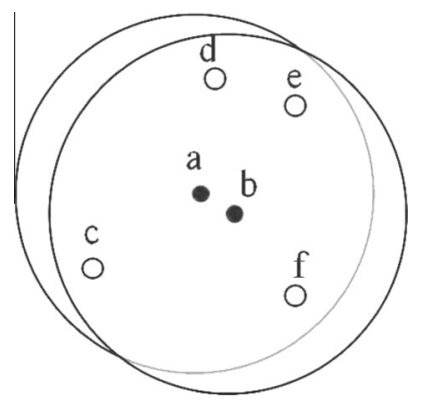

Fig. 8. Example of the neighboring set of nodes $a$ and $b$, which constitute the redundant set $R D=\{a, b\}$. The circles are centered at nodes $a$ and $b$, and have a radius equal to the transmission range $R$.

based on the indicator function in a network of 272 sensors, as arranged at random in Fig. 9. The goal is to show the effect of distributed characteristics on the global performance of management scheme in the large-scale WSN and validate the performance of proposed algorithm presented earlier. The top panel of Fig. 10 shows that 27 nodes (represented by green squares) among a total of 272 nodes (represented by red circles) got the chance to sleep during the first round by implementing our proposed algorithm, while the lower panel shows that other 27 nodes' (represented by blue squares) turn to sleep is the next round. We conclude that there are total 54 nodes, about $20 \%$ of nodes, to be scheduled in the distributed way to sleep.

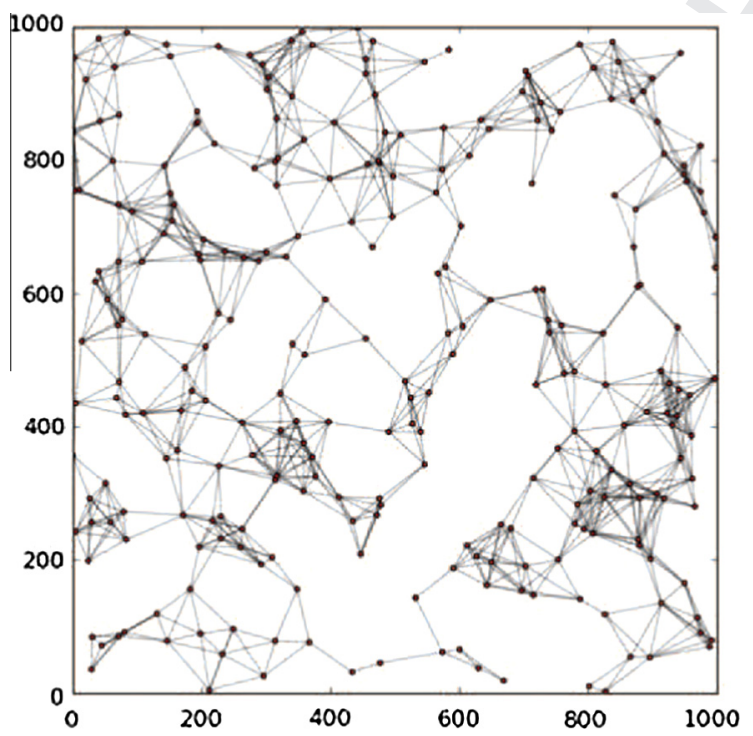

Fig. 9. Example of the original network for $R=100 \mathrm{~m}$.
Furthermore, we found that all the nodes that are scheduled to sleep do not break the connectivity and coverage conditions. It means that our proposed algorithm maintains the global performance of large-scale WSNs, while prolonging the network lifetime by scheduling part of nodes into the sleep mode in the distributed way. When the strict criterion of finding $R D \mathrm{~s}$ is relaxed, we validate that much more than $20 \%$ of nodes will get the chance to sleep by periodic rotation.

\subsection{Scalability}

The previous section described our implementation of a distributed management scheme and used it to demonstrate the performance of the schedule among $n b s$ in the $R D$ sets. In this section, our focus is on evaluating the scalability of the proposed management scheme to demonstrate its applicability to the large-scale WSNs. We
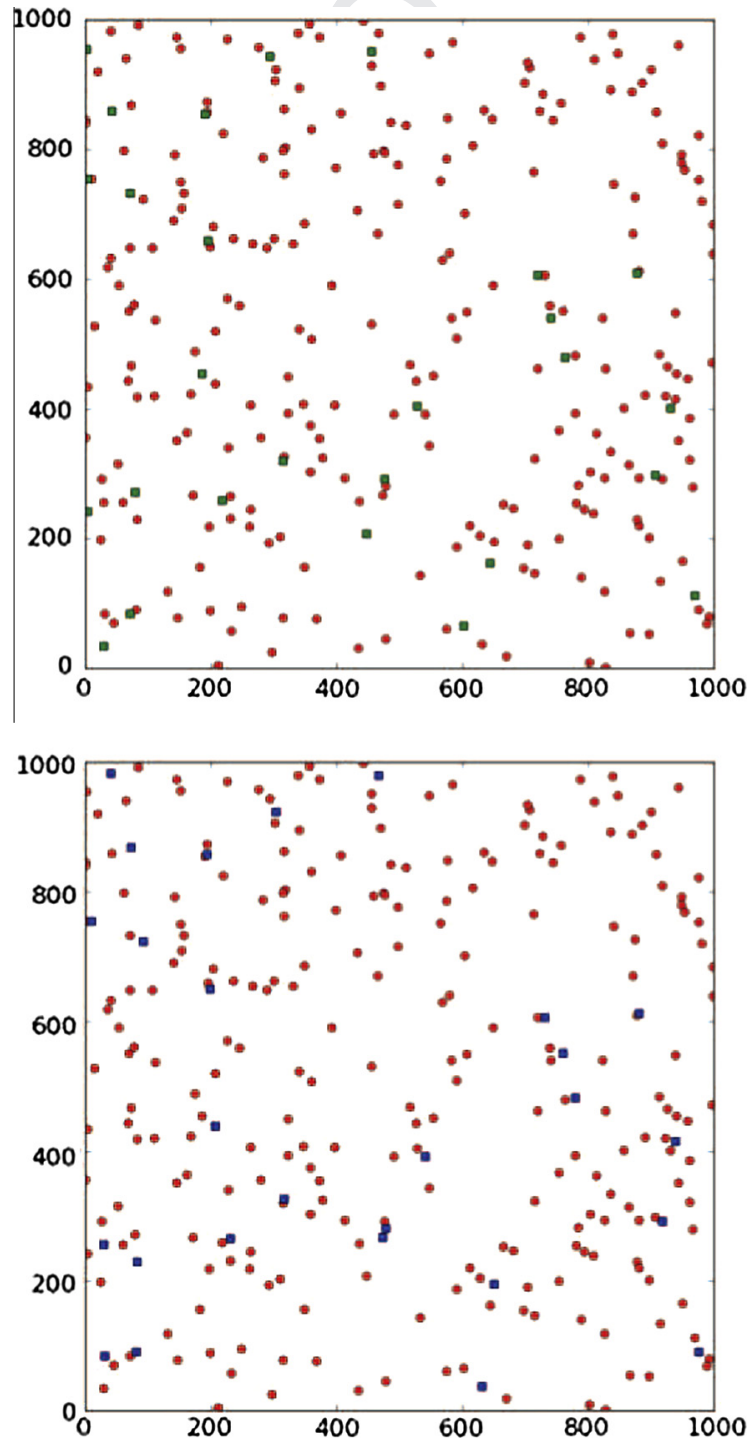

Fig. 10. ARD selection in a given network during the first round (top panel) and during the second round (lower panel). 


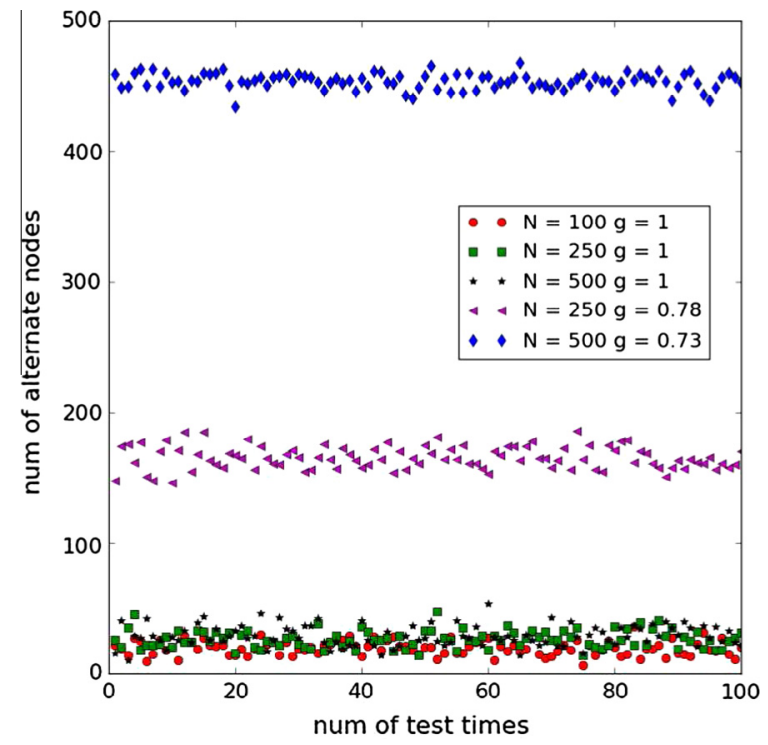

Fig. 11. Performance comparison of distributed management schemes on different scales of wireless sensor network and different $g$.

consider the WSNs for different scales, each node with an initial energy of $E$ units, the same sensing and transmission radii of 150 units, and deployed uniformly at random in a $1000 \times 1000$ units target field.

Recall that the $R D$ consists of nodes with the same $N B$. In order to improve the proposed scheme's performance further, we want to relax such a strict criterion of finding $R D$ s. First, the extent of overlapping between two NBs is evaluated by the percentage of duplicate nodes in these two, denoted by $g$. Then, the previous criterion is relaxed from $g=100 \%(g=1)$ to the extent that the given configuration of $g$ does not break the connectivity and coverage conditions of the network. We fix the values of $N$ and $g$ and plot, in Fig. 11, the number of alternate nodes by running the tests from 1 to 100 times. The plot shows that the number of alternate nodes achieved by our proposed distributed scheme is roughly the same for different tests under the fixed $N$ and $g$. This suggests that a stable number of alternate nodes can be attained by our proposed distributed management scheme. Thus, in practice, the sensors need not exchange any global information to ensure a sufficient number of alternate nodes without reducing the

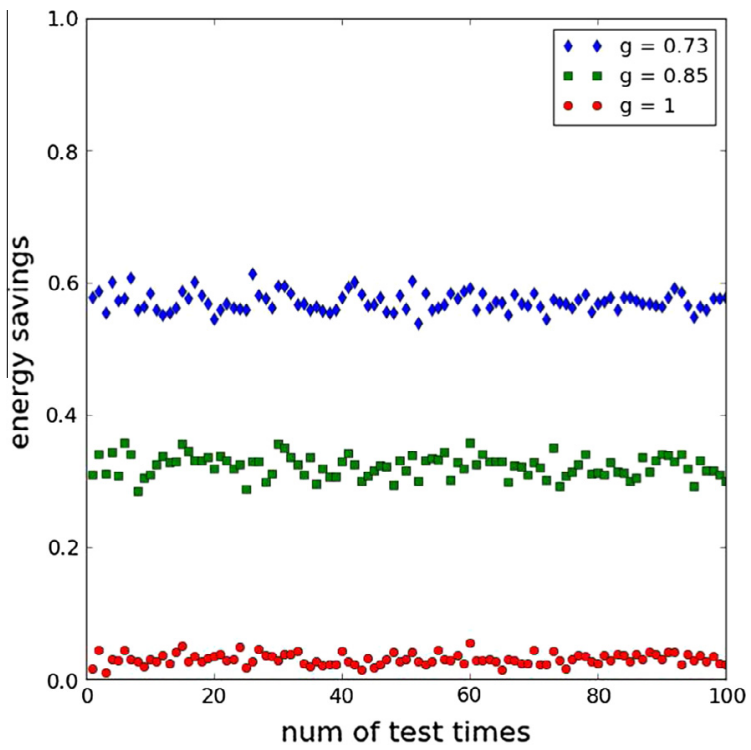

Fig. 13. Energy savings comparison of distributed management scheme under different configurations of $g$ in the given network.

global performance of WSN. When $g=1$, the larger the number of sensor present (here 500 within the area of $1000 \times 1000$ units), the more difficult becomes to find the completely same $N B$ among neighboring nodes. Therefore, as shown in Fig. 11, the number of alternate nodes have not been increased significantly for the large scales. However, when the strict criterion of finding $R D$ (i.e., $g=1$ ) is relaxed as low as $g=0.73$, ensuring the global performance (without breaking the connectivity and coverage conditions), Fig. 11 shows that the performance improves over the case of $g=1$ due to potential $R D$ s induced by the decreased $g$. Especially in the case of 500 nodes with $g=0.73$, the number of alternate nodes reaches around 450 , meaning that almost the whole number of deployed nodes can be set into a sleeping mode without decreasing the global performance of the network.

Next, we set up the random topology shown in the left panel of Fig. 12. We plot the ARD selection in the given WSN of 250 nodes for two different $g$ configurations. The middle panel shows that 99 nodes (represented by green squares) among the total of 250 nodes can be set into a sleeping mode during the first round and the total of 162
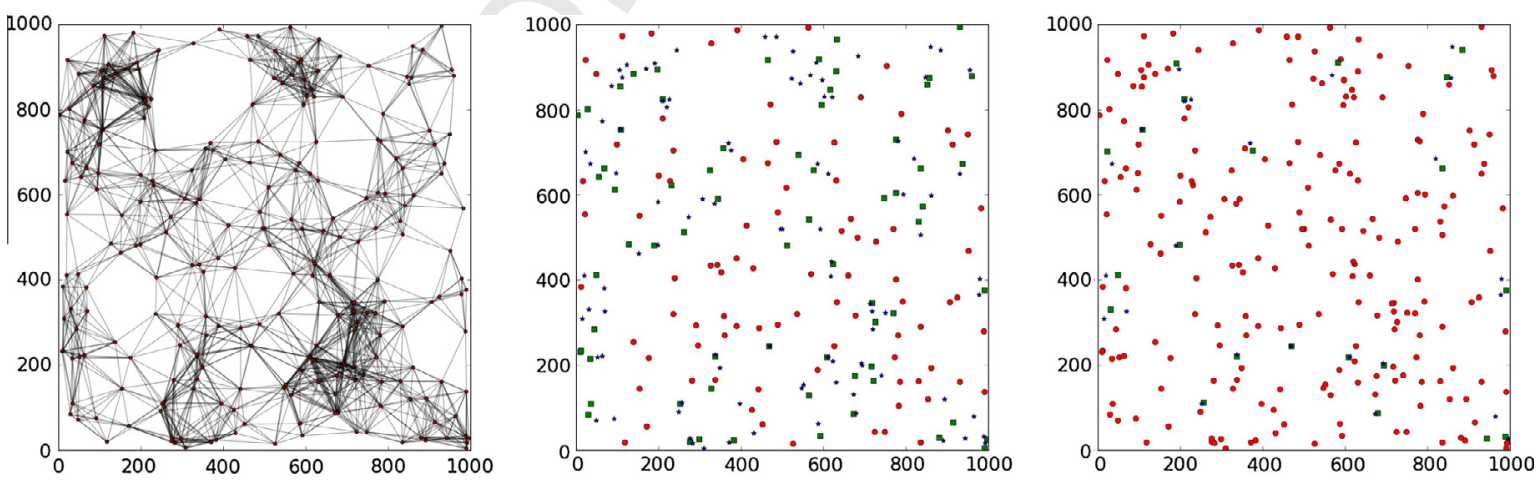

Fig. 12. $A R D$ selection in the given network (left panel) with $g=0.78$ (middle panel) and $g=1$ (right panel). 
nodes (represented by blue stars) can be alternated by implementing our proposed scheme and changing the $g$ from 1 to 0.78 . The right panel shows that, when $g=1$, 21 nodes (represented by green squares) among 250 nodes can be alternated during the first round and the total of 45 nodes (represented by blue stars) can be scheduled to sleep. Thus, in practice, through relaxing the value of $g$ to the extent that the changing configuration does not break the connectivity and coverage condition of the network, the sensors need only exchange the local information (sharing the neighboring information with its neighbors) to prolong the network lifetime by alternating the node to sleep. Furthermore, the number of alternate nodes increases to almost the total number of deployed nodes with the increase of deployed scale, meaning that our proposed management scheme shows good scalability. Note that, the larger the scale of WSN increases, the lower the parameter $g$ becomes without breaking the global conditions. Therefore, when a large-scale WSN is deployed, $g$ can be assigned a larger value for the smaller-scale network case to avoid the risk of requiring the global information.

As noted earlier in our performance evaluation, we compare the performance of our distributed management scheme between different configurations of $g$. We are interested in answering the following question: How much energy does our distributed management scheme save? To answer this question, we perform the following set of experiments. We randomly deploy 500 nodes in a $1000 \times 1000$ units target field, each with the same initial energy and the same sensing and transmission radii of 150 units. We fix the values of $g$ and plot energy savings by running the tests, from 1 and 100 times, in Fig. 13. We can see that our proposed scheme with $g=1$ reduces the average energy consumption by $5 \%$. When the strict criterion of $g$ is relaxed to be $85 \%$, the extent of energy saving is about $30 \%$. If the value of $g$ is relaxed as low as $g=0.73$ ensuring the global performance (without breaking the connectivity and coverage conditions), the extent of energy saving reaches about 60\%. Therefore, it becomes interesting how to select the proper configuration of $g$ to minimize the energy consumption without breaking the connectivity and coverage in the given WSN.

\section{Conclusions and future work}

We have discussed a wireless random sensor network model in which sensors are randomly deploy within a surface area $L^{2}$. The deployment of sensors is stopped when the whole network becomes fully connected for the first time, determining the number $N$ of total sensors required. We have obtained analytically an approximate mean value for $N$ which agrees remarkably well with the numerical results. We have studied several graph metric properties of the networks such as mean shortest path, mean clustering coefficient, mean node degree, mean maximum degree and the node degree distribution functions. The results suggest possible ways for obtaining optimized networks and further results are presented regarding a management protocol aimed at saving energy consumption and more efficient network functionality, by keeping full connectivity and above $90 \%$ coverage, without the need to consider cooperative features between neighboring nodes. For large scale networks, the assumption of a single sink node might not be feasible for implementing efficient management protocols. In those cases, the whole network could be partitioned into smaller units, each one controlled by a single sink. Then, the present results can be applied on a local basis, that is, for each sub-network in the system. We proposed a distributed management scheme to schedule the part of sensors to sleep by exchanging the neighboring information with their neighboring nodes, while the connectivity and coverage conditions are maintained. We also demonstrated that our proposed management scheme can deal with the large-scale WSN case by relaxing the $g$. Further, the simulation results show that our proposed scheme offers significant gains in number of alternate nodes as the deployed scale of network becomes larger when the configuration of $g$ is relaxed.

\section{Uncited reference}

[48].

\section{References}

[1] H. Wang, Y. Yang, M. Ma, Network lifetime global optimization by duality approach in wireless sensor networks, in: Proc. GLOBECOM 2007, Washington, DC, November 26-30, 2007.

[2] G.J. Pottie, W.J. Kaiser, Wireless integrated network sensors, Commun. ACM 43 (5) (2000) 51-58

[3] A. Cerpa, J. Elson, M. Hamilton, J. Zhao, D. Estrin, L. Girod, Habitat monitoring: application driver for wireless communications technology, in: Proc. Workshop on Data communication in Latin America and the Caribbean, SIGCOMM: ACM, ACM Press, New York, NY, 2001, pp. 20-41.

[4] L.F. Akyildiz, W. Su, Y. Sankarasubramaniam, E. Cayirci, A survey on sensor networks, IEEE Commun. Mag. (2002).

[5] A.J. Goldsmith, S.W. Wicker, Design challenges for energyconstrained ad hoc wireless networks, IEEE Wireless Commun. Mag. (2002).

[6] N.M. Freris, H. Kowshik, P.R. Kumar, Fundamentals of large sensor networks: connectivity, capacity, clocks, and computation, Proc. IEEE 98 (11) (2010).

[7] F. Ye, H. Luo, J. Cheng, S. Lu, L. Zhang, A two-tier data dissemination model for large-scale wireless sensor networks, in: ACM MOBICOM'02, Atlanta, Georgia, 2002.

[8] C. Lu, B.M. Blum, T.F. Abdelzaher, J.A. Stankovic, T. He, RAP: a realtime communication architecture for large-scale wireless sensor networks, in: IEEE Real-Time and Embedded Technology and Applications Symposium, 2002.

[9] Z. Yun, X. Bai, D. Xuan, T.H. Lai, W. Jia, Optimal deployment patterns for full coverage and $k$-connectivity $(k \leqslant 6)$ wireless sensor networks, IEEE Trans. Network. 18 (3) (2010).

[10] Xiangyang Li, Wireless Ad Hoc and Sensor Networks, Cambridge University Press, 2008.

[11] R. Madan, S. Cui, A.J. Goldsmith, Cross-layer design for lifetime maximization in interference-limited wireless sensor networks, IEEE Trans. Wireless Commun. 5 (11) (2006).

[12] J.-H. Chang, L. Tassiulas, Maximum lifetime routing in wireless sensor networks, IEEE/ACM Trans. Network. 12 (4) (2004).

[13] R. Madan, S. Lall, Distributed algorithms for maximum lifetime routing in wireless sensor networks, IEEE Trans. Wireless Commun. 5 (8) (2006).

[14] A. Giridhar, P.R. Kumar, Maximizing the functional lifetime of sensor networks, in: IPSN, 2005

[15] Guanrong Chen, Complex dynamical networks: an introduction, in: Lecture Notes in EE 6605 in City University of Hong Kong, 2007.

[16] H. Cai, X. Jia, M. Sha, Critical sensor density for partial connectivity in large area wireless sensor networks, ACM Trans. Sensor Network. 7 (4) (2011). 
[17] P. Wang, Z. Sun, M.C. Vuran, M.A. Al-Rodhaan, A.M. Al-Dhelaan, I.F. Akyildiz, On network connectivity of wireless sensor networks for sandstorm monitoring, Comput. Netw. 55 (5) (2011).

[18] P. Erdös, A. Rényi, On the evolution of random graphs, Publ. Math. Inst. Hung. Acad. Sci. 5 (1960) 17-60.

[19] B. Bollobas, Random Graphs, Cambridge Univ. Press, Cambridge, UK, 2001.

[20] E.N. Gilbert, Random plane networks, J. Soc. Indust. Appl. Math. 9 (1961) 533-543.

[21] P. Gupta, P.R. Kumar, Critical power for asymptotic connectivity in wireless networks, in: W.H. Fleming, W.M. McEneany, G. Yin, Q. Zhang (Eds.), Stochastic Analysis, Control, Optimization, and Applications: A Volume in Honor, Birkhauser, Boston, MA, 1998, pp. 547-566.

[22] M. Penrose, Random Geometric Graphs, Oxford Univ. Press, Oxford, UK, 2003.

[23] S. Misra, S.D. Hong, G. Xue, J. Tang, Constrained relay node placement in wireless sensor networks: formulation and approximations, IEEE Trans. Network. 18 (2) (2010).

[24] N. Bambos, Toward power-sensitive network architectures in wireless communications: concepts, issues and design aspects, IEEE Pers. Commun. Mag. 5 (1998) 50-59.

[25] R. Ramanathan, R. Rosales-Hain, Topology control of multihop wireless networks using transmit power adjustment, in: Proc. IEEE Infocom 2000, March 2000.

[26] W.I. Chen, N.F. Huang, The strongly connecting problem on multihop packet radio networks, IEEE Trans. Commun. 37 (1989) 293-295.

[27] R. Rajaraman, Topology control and routing in ad hoc networks: a survey, ACM Newslett. 33 (2) (2002) 60-73.

[28] L. Li, J. Halpern, V. Bahl, Y.-M. Wang, R. Wattenhofer, Analysis of a cone-based distributed topology control algorithms for wireless multi-hop networks, in: Proceedings of ACM Symposium on Principles of Distributed Computing, August 2001, pp. 264-273.

[29] V. Rodoplu, T. Meng, Minimum energy mobile wireless networks, IEEE J. Selec. Areas Commun. 17 (8) (1999) 1333-1344.

[30] Y. Wang, X.-Y. Li, Distributed spanner with bounded degree for wireless ad hoc networks, in: Parallel and Distributed Computing Issues in Wireless Networks and Mobile Computing, April 2002.

[31] R. Wattenhofer, L. Li, P. Bahl, Y.-M. Wang, Distributed topology control for power efficient operation in multihop wireless ad hoc networks, in: Proceedings of IEEE Infocom, 2001

[32] K. Alzoubi, X.-Y. Li, Y. Wang, P.-J. Wan, O. Frieder, Geometric spanners for wireless ad hoc networks, IEEE Trans. Parallel Distrib. Process. 14 (2003) 408-421.

[33] X.-Y. Li, Algorithmic, geometric, and graph issues in wireless networks, Wiley Wireless Communications and Mobile Computing (WCMC), Wiley, New York, 2002.

[34] K.M. Alzoubi, Virtual Backbone in Wireless AdHoc Networks, Ph.D. Dissertation, Illinois Institute of Technology, 2002.

[35] P.-J. Wan, K.M. Alzoubi, O. Frieder, Distributed construction of connected dominating set in wireless ad hoc networks, IEEE INFOCOM (2002).

[36] S. He, J. Chen, Y. Sun, Coverage and connectivity in duty-cycled wireless sensor networks for event monitoring, IEEE Trans. Parallel Distrib. Syst. 23 (3) (2012).

[37] S. Meguerdichian, F. Koushanfar, M. Potkonjak, M. Srivastava, Coverage problems in wireless ad-hoc sensor networks, IEEE Infocom 20013 (2001) 1380-1387.

[38] K. Kar, S. Banerjee, Node placement for connected coverage in sensor networks, in: Proceedings of the Workshop on Modeling and Optimization in Mobile, Ad Hoc and Wireless Networks (WiOpt'03), Sophia Antipolis, France, 2003.

[39] X. Bai, Z. Yun, D. Xuan, T. Lai, W. Jia, Optimal patterns for fourconnectivity and full coverage in wireless sensor networks, IEEE Trans. Mob. Comput. 9 (2010) 435-448.

[40] M. Hefeeda, M. Bagheri, Randomized $k$-coverage algorithms for dense sensor networks, in: Proc. of IEEE INFOCOM 2007 Minisymposium, Anchorage, AK, May 2007, pp. 2376-2380.

[41] M. Cardei, J. Wu, Energy-efficient coverage problems in wireless adhoc sensor networks, Comput. Commun. 29 (2006) 413-420.

[42] Guoliang Xing, Xiaorui Wang, Yuanfang Zhang, Chenyang Lu, Robert Pless, Christopher Gill, Integrated coverage and connectivity configuration for energy conservation in sensor networks, ACM Trans. Sensor Networks (TOSN) 1 (2005) 36-72.

[43] B. Carbunar, A. Grama, J. Vitek, O. Carbunar, Redundancy and coverage detection in sensor networks, ACM Trans. Sensor Networks (TOSN) 2 (2006) 94-128.

[44] Z. Zhou, S. Das, H. Gupta, Connected $k$-coverage problem in sensor networks, in: Proc. of International Conference on Computer Communications and Networks (ICCC'04), Chicago, IL, October 2004.

[45] A. Okabe, B. Boots, K. Sugihara, Spatial Tessellations Concepts and Applications of Voronoi Diagrams, Wiley, New York, 1992.

[46] P. Gupta, P.R. Kumar, The capacity of wireless networks, IEEE Trans. Network. 46 (2000) 388-404.

[47] H.E. Roman, A continuum percolation model for dispersed ionic conductors, J. Phys.: Condens. Matter 2 (1990) 3909.

[48] S. Dixit, E. Yanmaz, Ozan K. Tonguz, On the design of self-organized cellular wireless networks, IEEE Commun. Mag. (2005) 86-93.

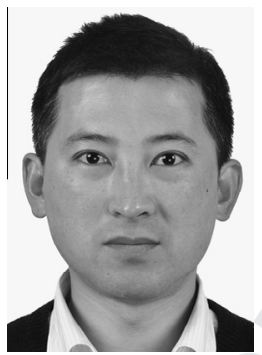

Hui Wang is currently university professor in the School of Mathematics, Physics, and Information Engineering at Zhejiang Normal University in China. He received the B.Eng. degree in 1998 and M.Eng. degrees in petroleum engineering in 2002 from Xi'an Shiyou University, Ph.D. degree at the School of Electronic, Information and Electrical Engineering in 2008 from Shanghai Jiao Tong University. He has been a postdoctoral scholar in ITCE division at Pohang University of science and technology (POSTECH) and Evry University in France. His research interests include optimization and control with applications in communication systems, wireless systems and data networking, with a current focus on information processing and resource management in ambient intelligence systems and large-scale wireless sensor networks.

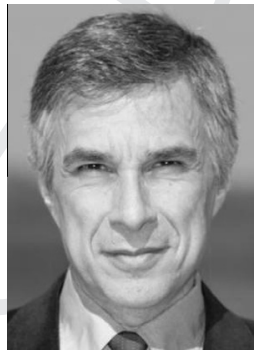

H. Eduardo Roman received the M.Eng. degree in Physics from University of Cuyo Bariloche, Argentina, in 1979 and Ph.D. degree in Physics from International School of Advanced Studies, Triest, Italy, in 1983. He is visiting professor in ITCE at Pohang University of Science and Technology in Korea. His research interests include modeling of complex systems, time series analysis from natural and social phenomena, and Financial Markets, Structural and Dynamical Properties of Linear Polymers, Electronic Structure of Nanostructured Materials (Fullerenes, Metal Clusters), Theory of Transport in Disordered Systems and Fractals.

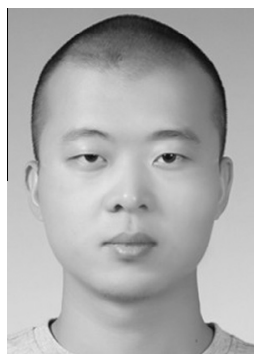

Yongfeng Huang received the B.Eng. degree in Computer Science from Xi'an Jiaotong University, Xi'an, China, in 2010, and the M.Eng. degree in IT Convergence Engineering from Pohang University of Science and Technology, Pohang, Korea, in 2012. He is currently working as a software engineer in www.dianping.com. His research interests include ubiquitous computing, smart home and human computer interaction. 